\title{
Réalisation d'une démarche d'analyse technologique par des élèves de science et technologie au secondaire : trois cas de figure
}

Jolyane Damphousse

Université du Québec à Trois-Rivières

Audrey Groleau

Université du Québec à Trois-Rivières

Ghislain Samson

Université du Québec à Trois-Rivières

\section{Résumé}

L'analyse technologique consiste à examiner un objet pour en comprendre le

fonctionnement. Dans cet article, nous étudions comment des élèves du secondaire

procèdent lorsqu'ils effectuent une démarche d'analyse technologique en classe de science et technologie. Les observations et entretiens réalisés auprès de douze élèves montrent

Canadian Journal of Education / Revue canadienne de l'éducation 44:3 (2021)

(C)2021 Canadian Society for the Study of Education/ Société canadienne pour l'étude de l'éducation 
qu'ils procèdent d'au moins trois manières différentes, que nous avons appelées « dissection séquentielle » (démonter l'objet en entier, puis tenter d'en comprendre le fonctionnement), « dissection par systèmes » (démonter l'objet partiellement pour comprendre l'articulation de ses systèmes) et « dissection en spirale » (démonter une pièce à la fois en tentant de comprendre sa fonction).

Mots-clés : analyse technologique, dissection mécanique, science et technologie

\begin{abstract}
Technological analysis involves examining an object to understand how it works. In this article, we study how high school students proceed while they perform a technological analysis in technology classes. Based on observations and interviews with twelve students, our results show that they use at least three different ways to do so, which we have labelled: sequential dissection (completely disassemble the object, then try to understand how it works), system-based dissection (partially disassemble the object to understand how its systems fit together), and circular dissection (disassemble one piece at the time and try to understand its function).
\end{abstract}

Keywords: technological analysis, mechanical dissection, science and technology 


\section{Introduction}

Les médias abordent de plus en plus le phénomène d'obsolescence, c'est-à-dire la tombée en désuétude, souvent prématurée, d'objets techniques, ce qui n'est pas sans conséquences économiques et environnementales. Prenons pour exemple le cas récent de l'action collective autorisée contre Apple au sujet de la lenteur de certains modèles d'iPhone. Une mise à jour du système d'exploitation produirait un ralentissement du téléphone qui le rendrait inutilisable, incitant les utilisateurs et utilisatrices à s'en procurer un nouveau (Gonzalez, 2019). Ce recours collectif, à l'instar de nombreux autres évènements similaires récents, ravive le débat entourant l'obsolescence programmée (Trudel, 2019), soit la réduction volontaire de la durée de vie d'un produit par le fabricant (Latouche, 2012).

Une façon efficace de faire face à l'obsolescence programmée est la réparation d'objets (Paré, 2018). Afin de la rendre accessible à tous et à toutes, plusieurs initiatives ont récemment été mises en place. Par exemple, les « Repair Cafés ${ }^{1}$ », qui existent partout à travers le monde, permettent aux citoyens et citoyennes d'apprendre comment réparer les objets auprès d'experts bénévoles. Les « $\mathrm{FabLab}^{2}$ » rendent quant à eux possible la réparation de certains objets dont une pièce est manquante ou brisée. En effet, ces ateliers mettent à la disposition des citoyens et citoyennes des outils de fabrication comme des imprimantes 3D. À l'aide de ces outils, ils peuvent concevoir la pièce brisée ou manquante et ainsi réparer leur objet.

L'obsolescence et la réparation d'objets trouvent leur place de différentes manières dans le Programme de formation de l'école québécoise (PFEQ) en science et technologie au secondaire. La problématique environnementale entourant les matières résiduelles est notamment proposée comme fil conducteur servant à contextualiser de nombreux savoirs prescrits des cours Science et environnement (Ministère de l'Éducation,

1 Liste des « Repair Cafés » (https://repaircafe.org/fr/visiter/, consulté le 5 janvier 2021).

2 Liste des «Fab Labs » (https://www.fablabs.io/labs, consulté le 5 janvier 2021). 
du Loisir et du Sport [MELS], 2007b) ${ }^{3}$ et Science et technologie de l'environnement (MELS, 2007c) en quatrième secondaire. Par ailleurs, le programme prescrit l'apprentissage de sept démarches scientifiques et technologiques. Si l'obsolescence peut être abordée à l'aide de la démarche de construction d'opinion (Groleau et Pouliot, 2015) ${ }^{4}$, l'angle d'entrée de la réparation d'objets par l'entremise de la démarche d'analyse technologique - l'idée d'observer un objet technique, souvent en le démontant, pour en comprendre le fonctionnement — peut aussi être exploité. Plus encore, la compétence disciplinaire « Mettre à profit ses connaissances scientifiques et technologiques » prévoit explicitement l'entretien et la réparation d'objets dans le cadre du cours Applications technologiques et scientifiques (MELS, 2007a) de la troisième et de la quatrième année du secondaire.

La réalisation d'une démarche d'analyse technologique, qui peut être plus ou moins approfondie selon le contexte, est préalable à la réparation de tout objet, puisque pour le réparer, il faut en comprendre le (dys)fonctionnement. On peut ainsi associer cette démarche aux visions démocratique et utilitariste de l'enseignement des sciences et de la technologie (Barma et Guilbert, 2006) : à la vision démocratique d'une part, puisque de savoir réparer des objets, voire de partager ce savoir avec d'autres, peut devenir une forme d'engagement citoyen dans la lutte à l'obsolescence ; à la vision utilitariste d'autre part, car la réparation d'objets techniques est aussi une manière de faire usage de ses connaissances technoscientifiques dans la vie quotidienne et de devenir une consommatrice ou un consommateur plus averti.

Dans cet article, basé sur le mémoire de maitrise de Damphousse (2017), nous nous intéressons à la démarche d'analyse technologique telle qu'elle est vécue en classe de science et technologie au secondaire au Québec.

3 À partir du deuxième cycle du secondaire, les élèves doivent choisir entre deux séquences considérées comme équivalentes, mais dont les méthodes d'enseignement diffèrent : Science et technologie (S\&T) ou Applications technologiques et scientifiques (ATS). Les élèves peuvent aussi réaliser un cours optionnel en quatrième secondaire leur permettant d'accéder aux cours de chimie et physique de cinquième secondaire. Pour les élèves de la séquence S\&T, le cours optionnel s'intitule Science et technologie de l'environnement, alors que pour les élèves de la séquence ATS, il porte le nom Science et environnement.

4 Il s'agit de se construire une opinion réfléchie et nuancée au sujet d'une problématique technoscientifique en prenant en considération ses aspects scientifiques, technologiques, économiques, éthiques, environnementaux, etc. 


\section{Démarche d'analyse technologique : quelques balises théoriques}

L'analyse technologique est une expression utilisée par le MELS dans les programmes issus de la vaste réforme du curriculum du début des années 2000. C'est en fait une expression générique qui regroupe plusieurs démarches inspirées de l'industrie dans le domaine de l'ingénierie, dont les principales sont la rétroconception, la reconception et la dissection mécanique. Le MELS (2007a) décrit l'analyse technologique comme suit :

L'analyse d'un objet technique ou d'un système technologique implique la reconnaissance de sa fonction globale, de façon à cerner le besoin auquel il répond. [...] L'examen des diverses composantes d'un objet technique ou d'un système technologique s'avère également nécessaire pour déterminer leurs fonctions respectives. (p. 30)

En réalisant un examen approfondi des documents ministériels, nous constatons que la dissection mécanique est la démarche qui y est privilégiée parmi toutes celles qui peuvent être associées à l'analyse technologique. La dissection mécanique implique de démonter et d'analyser l'objet, pour ensuite, possiblement, le remonter; chacun de ces éléments est d'ailleurs mentionné dans le programme de formation. Alors que la rétroconception exige de plus la création d'une représentation en fin de processus, la reconception est utilisée dans une optique d'amélioration d'un produit pour le rendre plus compétitif. Ces deux derniers éléments ne figurent pas explicitement dans le PFEQ, et c'est la raison pour laquelle nous avons spécifiquement invité les élèves participant à cette recherche à réaliser une dissection mécanique.

Cette démarche « consiste à démonter un produit afin de voir comment il fonctionne et à quoi sert chacune des composantes » (Doucet et al., 2007, p. 3). Bien que les auteurs s'entendent pour dire que la dissection mécanique demande de démonter et d'analyser l'objet (Dalrymple, 2009 ; Dalrymple et al., 2011 ; Doucet et al., 2007 ; Younis et Tutunji, 2012), tous n'abordent pas l'étape du remontage. Dalrymple (2009), qui envisage cette démarche comme une activité de type DAA (Disassemble/Analyze/ Assemble), inclut explicitement la possibilité que l'élève remonte l'objet après l'avoir démonté et analysé. En dépit du portrait très linéaire de la dissection mécanique brossé par ces définitions, cette démarche est itérative et elle inclut aussi des temps d'observation. 
La dissection mécanique requiert ainsi l'intervention d'une autre démarche : la démarche d'observation (Dalrymple, 2009 ; Doucet et al., 2007 ; Elizalde et al., 2008). Alors que Dalrymple (2009) mentionne que la dissection mécanique est une occasion de développer la capacité d'observation, Doucet et al. utilisent le verbe « voir » dans leur définition de la dissection mécanique : « [...] voir comment [l'objet] fonctionne [...]» (2007, p. 3, nous soulignons). En outre, l'observation de l'objet technologique permet de reconnaitre et d'inventorier chacune de ses composantes. L'observation, tout comme l'analyse technologique (dont la dissection mécanique fait partie) ou la construction d'opinion, est l'une des sept démarches que les élèves doivent apprivoiser au deuxième cycle du secondaire (MELS, 2007a, 2007b).

L'analyse technologique n'est pas employée qu'à l'école : elle constitue aussi une démarche bien connue et documentée dans l'industrie. Notons, par exemple, la taxonomie de l'ingénierie inversée (reverse engineering) - l'idée étant ici plus précisément de démonter un objet pour en comprendre le fonctionnement et le refaire d'une façon améliorée — de Chikofsky et Cross (1990). Il s'agit d'une source importante de définitions autour de différentes déclinaisons de l'analyse technologique qui ont été citées à de multiples reprises ${ }^{5}$. Connaissant sa place dans l'industrie, il n'est pas étonnant de constater qu'il existe divers textes au sujet de l'enseignement et de l'apprentissage de l'analyse technologique et de la dissection mécanique dans les écoles supérieures d'ingénierie (Dalrymple, 2009 ; Dalrymple et al., 2011 ; Elizalde et al., 2008 ; Sandborn et al., 2009 ; Younis et Tutunji, 2012). Du côté de l'enseignement secondaire, les balises ministérielles relatives à l'analyse technologique (MELS, 2007a, 2007b) sont plutôt claires, voire assez détaillées. Or, très peu de recherches ont été réalisées au sujet de la dissection mécanique telle qu'elle est enseignée ou apprise en classe de science et technologie au Québec. Les écrits à ce sujet, qui concernent les ordres d'enseignement primaire et secondaire, sont surtout des recueils de conseils ou des exemples d'activités. À notre connaissance, il n'existe aucune recherche qui répertorie les manières dont des élèves du secondaire opérationnalisent cette démarche. Dans cet article, nous répondons à la question de recherche suivante : de quelles façons des élèves du secondaire en science et technologie procèdent-ils lorsqu'ils réalisent une dissection mécanique?

5 Cité à près de 3000 reprises en date du 28 aout 2019 selon Google Schoolar. 


\section{Méthodologie}

La recherche présentée dans cet article est issue du mémoire de maitrise de la première autrice, sous la supervision des deuxième et troisième auteurs. Nous avons réalisé une étude exploratoire de nature qualitative descriptive pour examiner les manières dont des élèves du secondaire opérationnalisent la dissection mécanique. Un échantillonnage non probabiliste par choix raisonné (Fortin et Gagnon, 2016) a permis de sélectionner douze participants et participantes de quatrième secondaire du profil Applications technologiques et scientifiques provenant de la région de la Mauricie ${ }^{6}$. En effet, non seulement les élèves de quatrième secondaire sont soumis à l'examen ministériel en Science et technologie, mais la dissection mécanique y occupe habituellement une part non négligeable. À titre d'exemple, en 2017-2018, $20 \%$ des points de l'examen y étaient réservés dans la séquence Science et technologie et $24 \%$ dans la séquence Applications technologiques et scientifiques (Ministère de l'Éducation et de l'Enseignement supérieur [MEES], 2016). Par ailleurs, les participants et participantes ont en commun d'être engagés dans la séquence Applications technologiques et scientifiques, et cela, puisque c'est celle qui amène les élèves à faire le plus d'analyses technologiques, et donc, de dissections mécaniques.

Nous avons réalisé la collecte de données en deux étapes : par l'observation filmée d'une dissection mécanique, puis avec une entrevue individuelle semi-dirigée. Nous avons d'abord demandé aux participants et participantes de réaliser une dissection technologique d'un diffuseur de fragrance automatique (voir Figure 1), c'est-à-dire de tenter d'en comprendre le fonctionnement. Le choix de cet objet s'explique par son niveau de complexité adéquat pour des élèves de quatrième secondaire, par le fait qu'il est facile à démonter et à remonter sans être abimé, parce que le risque de blessure pendant les manipulations est minime, et parce qu'il permet de solliciter différents sens des élèves ${ }^{7}$. De plus, il contient des systèmes de transmission et de transformation du mouvement, un circuit électrique, un moteur, et ainsi de suite. À ce moment, les élèves,

6 Les participants et participantes proviennent de cette région pour des raisons de proximité avec l'université d'attache des membres de l'équipe de recherche.

7 Le deuxième objectif du mémoire, non traité dans cet article, est de décrire de quelles façons les élèves du secondaire en science et technologie utilisent leurs sens lors de situations d'observation, et plus précisément pendant une dissection mécanique. 
en équipe de deux personnes (six équipes au total), ont démonté l'objet et expliqué son fonctionnement oralement. Durant l'activité, l'étudiante-chercheuse a observé la démarche des élèves en prenant des notes dans un journal de bord. De plus, nous avons réalisé un enregistrement audiovisuel de l'activité.

\section{Figure 1}

\section{Diffuseur de fragrance automatique}

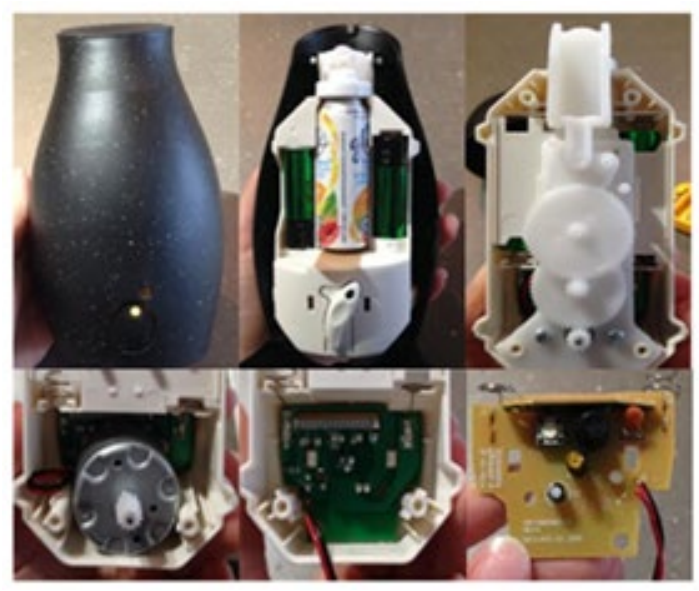

Un délai de quelques jours a été prévu entre la première et la deuxième étape de la collecte des données. Une analyse préliminaire de l'enregistrement audiovisuel ainsi que du journal de bord nous a permis de formuler les questions plus précises de l'entretien semi-dirigé, qui visait à permettre à chaque élève d'expliciter la manière dont les membres de l'équipe ont procédé lors de leur dissection mécanique. L'étudiantechercheuse a rencontré un à un tous les participants et participantes. Des questions très similaires ont été posées aux membres d'une même équipe. Une certaine triangulation entre les réponses des membres d'une même équipe était alors possible.

Au chapitre de l'analyse, nous avons d'abord rédigé une description détaillée de chaque enregistrement audiovisuel ainsi que de chaque entretien semi-dirigé. Pour ce faire, nous avons effectué des lectures et des visionnements répétés, rédigé des notes et un résumé reprenant des narrations des participants et participantes en nous inspirant des étapes de l'examen phénoménologique de Paillé et Mucchielli (2016). À partir de ces résumés, nous avons conçu des tableaux récapitulatifs des étapes de la dissection 
mécanique réalisée par chacune des équipes. Une fois cette première analyse terminée, nous avons procédé à une analyse à l'aide des catégories conceptualisantes (Paillé et Mucchielli, 2016), ce qui nous a permis d'obtenir trois cas de figure relatifs à la réalisation de la dissection mécanique, c'est-à-dire trois manières distinctes de procéder pour les participants et participantes que nous avons pu observer.

\section{Résultats : trois cas de figure relatifs à la dissection mécanique en classe de science et technologie au secondaire ${ }^{8}$}

En comparant les tableaux récapitulatifs des six équipes, trois cas de figure ont émergé à partir de nos analyses concernant la démarche utilisée par les participants et participantes lorsqu'ils réalisent une dissection mécanique. Ces cas de figure sont ainsi nommés : « dissection mécanique séquentielle », « dissection mécanique par systèmes » et « dissection mécanique en spirale ».

\section{Dissection mécanique séquentielle}

L'analyse de la démarche réalisée par les élèves lors de la dissection mécanique nous a permis de constater que trois équipes ( $\mathrm{A}, \mathrm{E}$ et $\mathrm{F}$ ) ont procédé selon des étapes similaires. Les étapes communes aux trois équipes ont été mises en évidence (cases grises) dans le Tableau 1, ce qui permet de bien distinguer les similitudes entre ces équipes, et cela, bien que leurs démarches, dans l'ensemble, ne soient pas totalement identiques.

8 Les résultats présentés dans cette section (tableaux, figures, définitions, propriétés et conditions d'existence) sont tirés tels quels du mémoire de maitrise de la première autrice. Les tableaux et figures apparaissent aussi dans Damphousse, J., Groleau, A. et Samson, G. (accepté). La dissection mécanique, à la fois démarche d'analyse technologique et d'observation. Partie 1 : analyse technologique. Spectre. 


\section{Tableau 1}

Comparaison des étapes de la dissection mécanique réalisée par les membres des équipes A, E et $F$

\section{Les étapes de la dissection Les étapes de la dissection Les étapes de la dissection mécanique réalisée par les mécanique réalisée par les mécanique réalisée par les membres de l'équipe $A$ membres de l'équipe $\mathbf{E}$ membres de l'équipe $\mathbf{F}$}

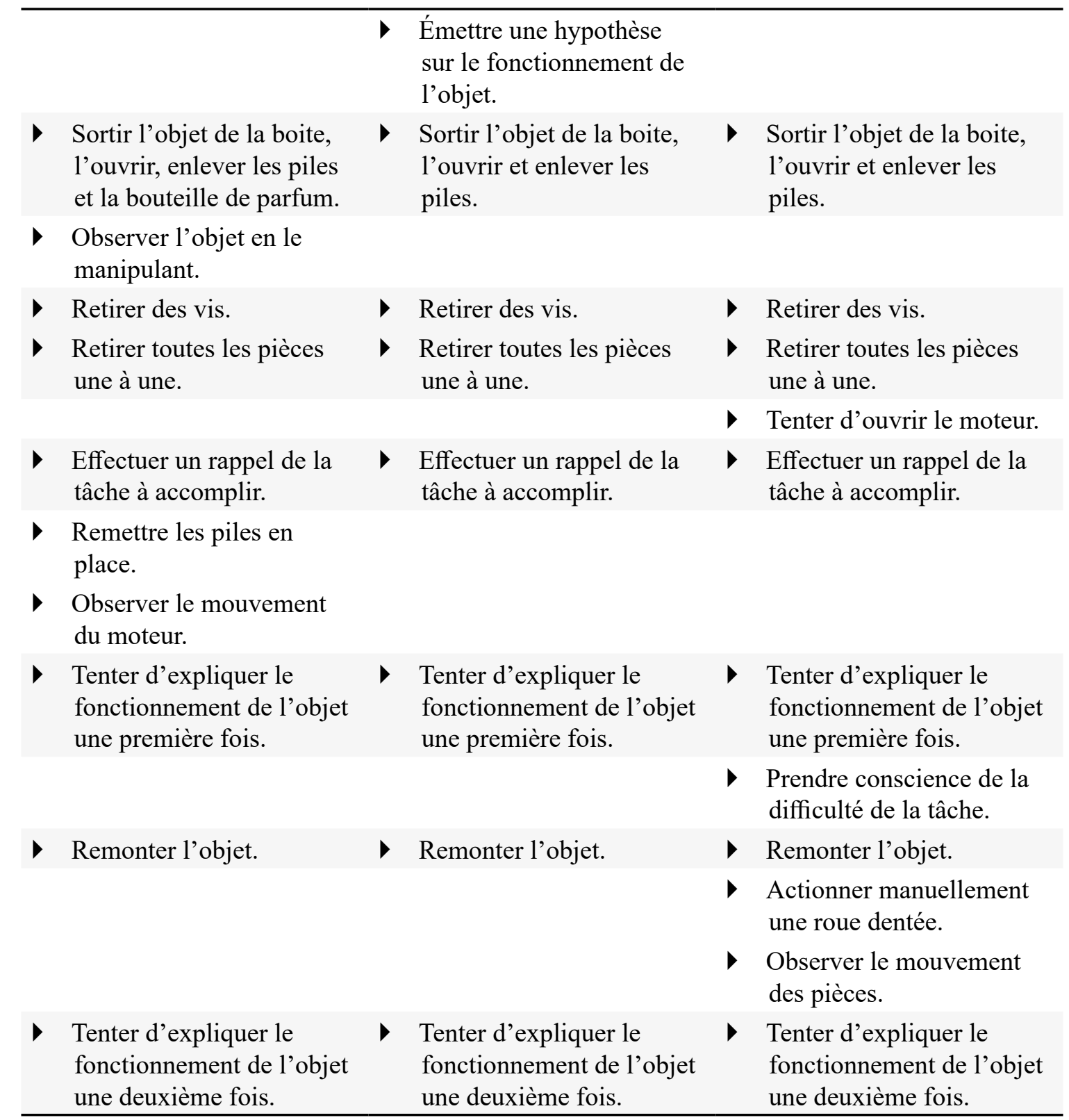


Lors de la conception de catégories conceptualisantes, il est primordial de les définir, d'identi $\neg$ fier leurs propriétés et leurs conditions d'existence ${ }^{9}$ (Paillé et Mucchielli, 2016). Ainsi, la dissection mécanique séquentielle se définit comme la dissection d'un objet selon une séquence dans le temps pendant laquelle l'élève ouvre d'abord l'objet et en retire toutes les pièces, remonte l'objet, puis en comprend le fonctionnement. Elle possède trois propriétés, soit le retrait de plusieurs ou de toutes les pièces dans un premier temps, le rappel de la tâche à accomplir dans un deuxième temps et le remontage de l'objet dans un troisième temps. Il est à noter que le rappel de la tâche à accomplir a été réalisé de façon explicite par les participants et participantes. Par exemple, durant la dissection mécanique, le participant 2 a mentionné : « attends, je pense que c'est ça qu'il faut savoir [en pointant la feuille de papier sur laquelle la tâche est inscrite] ». Comme conditions d'existence, cette catégorie demande de réaliser la dissection d'un objet et de faire un effort de compréhension de son fonctionnement. De plus, le démontage de l'objet et la compréhension de son fonctionnement sont réalisés à deux moments distincts. Le schéma suivant illustre l'idée selon laquelle le démontage et la compréhension du fonctionnement sont séparés dans le temps par le rappel de la tâche à accomplir. De plus, lors de la dissection mécanique séquentielle, l'élève n'a pas d'autre choix que de remonter l'objet et de l'observer afin de comprendre son fonctionnement.

\section{Figure 2}

\section{Illustration de la dissection mécanique séquentielle}

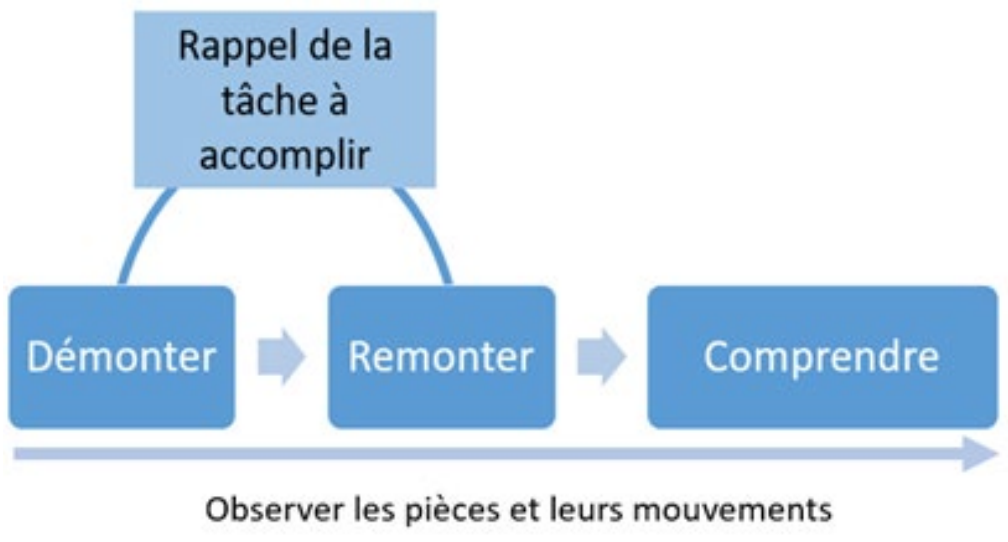

9 Une condition d'existence réfère à toute situation, expérience ou tout évènement en l'absence desquels le phénomène n'existe pas (Paillé et Mucchielli, 2016). 


\section{Dissection mécanique par systèmes}

Deux équipes ont réalisé une dissection mécanique que nous avons appelée « par systèmes ». Dans le Tableau 2, les différentes étapes réalisées par ces deux équipes sont exposées. Rappelons que les étapes comparables apparaissent dans des cellules grises. Dans ce cas de figure, certaines des étapes comparables ont été réalisées dans un ordre différent par les deux équipes. Ainsi, pour les faire ressortir, nous avons utilisé l'orange et le vert plutôt que le gris.

\section{Tableau 2}

Comparaison des étapes de la dissection mécanique réalisée par les membres des équipes $B$ et $D$

\section{Les étapes de la dissection mécanique réalisée par les membres de l'équipe $B$}

- Sortir l'objet de la boite et l'ouvrir.

- Retirer des vis.

- Observer l'objet.

- Actionner le bouton involontairement.

- Retirer des pièces contenues dans l'objet par regroupement (par systèmes).

- Observer des pièces de l'objet.

- Remonter l'objet.

- Actionner manuellement le mécanisme.

- Observer le mouvement des pièces.

- Expliquer le fonctionnement de l'objet.
Les étapes de la dissection mécanique réalisée par les membres de l'équipe $D$

- Sortir l'objet de la boite.

- Actionner le bouton volontairement.

- Retirer les piles et la bouteille de parfum.

- Retirer des vis.

- Actionner manuellement une roue dentée.

- Observer le mouvement des pièces.

- Retirer des roues dentées en les gardant regroupées (par systèmes).

- Tenter d'expliquer le fonctionnement de l'objet.

- Tenter de remonter l'objet.

- Échapper des pièces sur le sol.

- Abandonner. 
Bien que l'une des équipes ait mené à bien la démarche complète et que l'autre équipe ait abandonné en cours de route, ces deux équipes ont en commun d'avoir retiré les pièces en les gardant regroupées par systèmes. Du côté de l'équipe $\mathrm{D}$, les participantes ont retiré le système de roues dentées du diffuseur et l'ont conservé ainsi regroupé, alors que du côté de l'équipe $B$, les élèves ont scindé l'objet en ses différents systèmes (p. ex. le système de roues dentées et le système électrique). Une fois l'objet démonté, les deux équipes ont tenté de remonter ou ont remonté l'objet afin d'arriver à bien comprendre son fonctionnement.

La dissection mécanique par systèmes se définit comme la dissection d'un objet dans laquelle l'élève ouvre d'abord l'objet, en retire les pièces en les conservant regroupées par systèmes (p. ex., le système de roues dentées, le système électrique), puis le remonte en recherchant la compréhension du fonctionnement de l'objet. Ce cas de figure possède deux propriétés, soit la conservation des pièces regroupées par systèmes et la nécessité de remonter l'objet. Au regard des conditions d'existence, cette catégorie demande nécessairement de réaliser la dissection d'un objet et de faire un effort de compréhension de son fonctionnement. Aussi, le démontage de l'objet doit être réalisé en conservant les pièces d'un même système regroupées. La figure suivante montre, grâce aux pièces de casse-tête qui représentent chacune un système, que l'on peut retrouver dans un objet quelconque l'idée de démonter l'objet en quelques regroupements de pièces. Outre le démontage, les élèves doivent aussi remonter l'objet et l'observer pour comprendre son fonctionnement.

\section{Figure 3}

Illustration de la dissection mécanique par systèmes

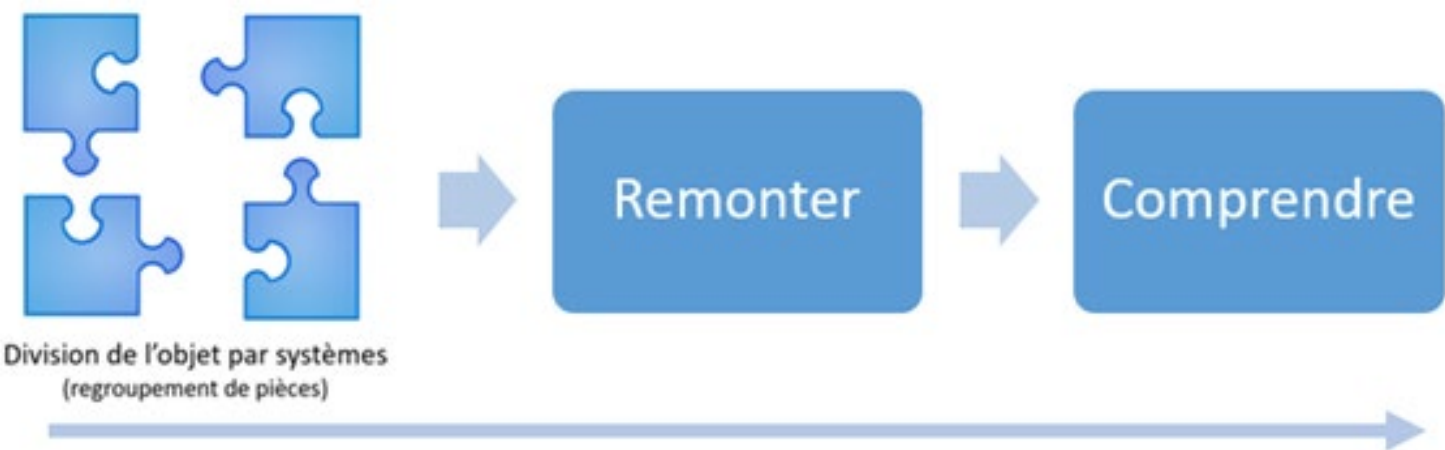

Observer les pièces et leurs mouvements 


\section{Dissection mécanique en spirale}

Ce cas de figure a été observé chez une seule équipe. En examinant le Tableau 3 qui décline les étapes de la démarche réalisée par l'équipe, il est possible de constater une certaine répétition. En effet, les membres de cette équipe ont enlevé des pièces, actionné volontairement le bouton de démarrage et observé l'objet à plusieurs reprises durant la dissection.

\section{Tableau 3}

Étapes de la dissection mécanique réalisée par les membres de l'équipe $C$

\section{Les étapes de la dissection mécanique réalisée par les membres de l'équipe $\mathbf{C}$}

Sortir l'objet de la boite, l'ouvrir et enlever les piles.

Retirer des vis.

Observer 1'objet.

Actionner le bouton volontairement à deux reprises.

Remettre les piles en place.

Actionner le bouton volontairement à quelques reprises.

Observer le mouvement des pièces.

Retirer les roues dentées.

Actionner le bouton volontairement à quelques reprises.

Observer le mouvement du moteur.

Expliquer le fonctionnement de l'objet.

Cette démarche a de particulier le fait que les élèves ont volontairement actionné le bouton de mise en marche de l'objet à plusieurs reprises tout au long de la dissection et qu'ils n'ont pas remonté l'objet. En fait, ils ont actionné le bouton, observé et retiré des pièces en alternance. La dissection mécanique en spirale se définit comme la dissection d'un objet dans laquelle l'élève met d'abord l'objet en marche, observe son mouvement et retire une ou des pièces, pour ensuite recommencer ces trois étapes autant de fois qu'il est nécessaire pour en arriver à une compréhension du fonctionnement de l'objet. Tout comme les deux autres cas de figure, la dissection mécanique en spirale revêt des propriétés et des conditions d'existence propres aux catégories conceptualisantes (Paillé et Mucchielli, 2016). Les propriétés de ce cas de figure sont les suivantes : la démarche combine la mise en marche de l'objet, l'observation et le retrait de pièces, et ces trois 
étapes doivent être effectuées à plusieurs reprises. Les conditions d'existence de cette catégorie sont de réaliser la dissection d'un objet, de faire un effort de compréhension du fonctionnement et de reprendre la même succession d'étapes $\neg$ à plusieurs reprises (mise en marche, observation, retrait de pièces). La Figure 4, par la schématisation de la spirale, montre l'itération entre les trois étapes.

\section{Figure 4}

Illustration de la dissection mécanique en spirale

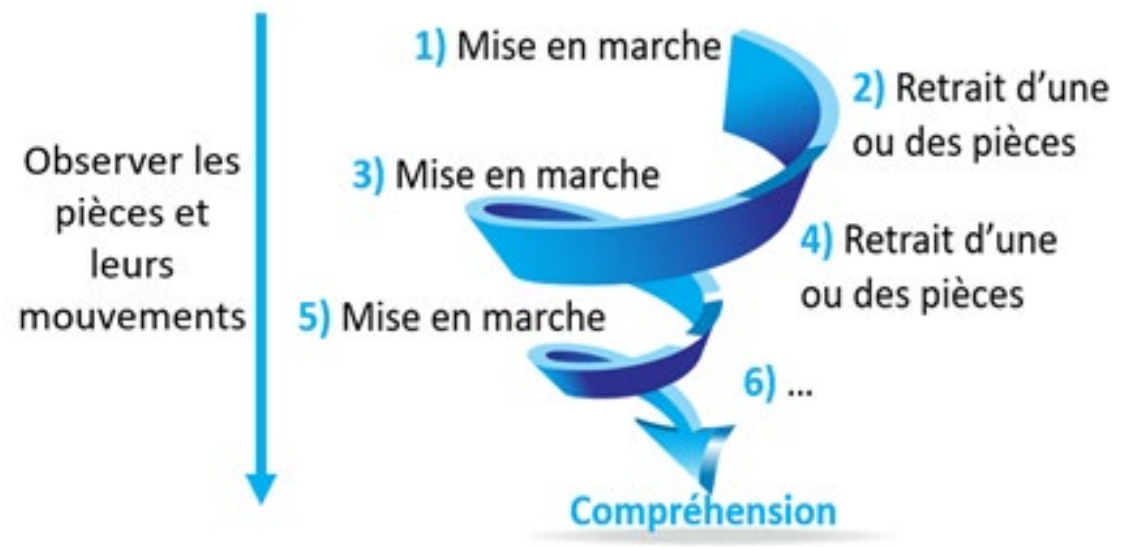

\section{Discussion}

Dans cette section, nous décrirons d'abord la démarche réalisée par les élèves lors de la dissection mécanique au regard de la définition de la dissection mécanique présentée dans les balises théoriques de cet article. Puis, nous discuterons de la démarche utilisée en fonction de l'objet proposé pour l'analyse et le rôle de la définition de la dissection mécanique pour le cas de figure séquentiel. Finalement, nous formulerons des recommandations pour l'enseignement de la dissection mécanique dans le cadre des cours de Science et technologie au secondaire.

\section{Description de la démarche utilisée par les participants}

Nous constatons que la dissection mécanique proposée demande nécessairement de démonter l'objet à l'étude. En effet, tous les élèves ont démonté le diffuseur de fragrance automatique lors de l'activité. Les définitions des différents auteurs s'accordent sur ce 
point : une dissection mécanique comporte nécessairement une étape de démontage (Dalrymple, 2009 ; Dalrymple et al., 2011 ; Doucet et al., 2007 ; Younis et Tutunji, 2012). Par ailleurs, tous les élèves ont dû analyser l'objet pour tenter de comprendre son fonctionnement. C'est pourquoi la dissection mécanique constitue une démarche de type « analyse technologique ». Un élément important différencie les équipes : 1'une d'entre elles n'a pas remonté l'objet, alors que toutes les autres l'ont fait. Rappelons que l'étape du remontage n'est pas identifiée par tous les auteurs comme faisant partie de la dissection mécanique. C'est dans la définition de Dalrymple (2009) que nous avons pu cerner cette étape. De plus, toutes les équipes ont eu recours à la démarche d'observation au cours de l'activité, ce qui confirme l'importance de la démarche d'observation dans la démarche d'analyse technologique (et donc, dans la dissection mécanique) telle qu'elle est soulignée dans les écrits recensés (Dalrymple, 2009 ; Doucet et al., 2007 ; Elizalde et al., 2008). Finalement, certains élèves ont employé une démarche itérative, alors que d'autres ont travaillé de façon plus linéaire.

\section{Choix de la démarche utilisée (dissection séquentielle, dissection par systèmes, dissection en spirale) en fonction de l'objet proposé}

La démarche utilisée par les élèves peut être influencée par le choix de l'objet à disséquer. Prenons le cas de figure de la dissection séquentielle, qui demande, du moins dans cette recherche, d'avoir la capacité de remonter entièrement l'objet afin d'arriver à bien comprendre son fonctionnement. Il est en effet très difficile de comprendre le fonctionnement d'un objet lorsqu'un grand nombre de pièces disparates (une vingtaine dans le cas du diffuseur de fragrance automatique) sont étalées sur une table ; il peut toutefois être aussi ardu de le remonter.

Lorsque des élèves réalisent la dissection mécanique d'un objet dont le fonctionnement est simple à leurs yeux, et notamment lorsqu'il comporte peu de pièces, il est possible que la dissection mécanique séquentielle soit une façon efficace de procéder. Prenons l'exemple d'un stylo pour des élèves de quatrième secondaire : ils n'auront aucune difficulté à le démonter, à comprendre son fonctionnement et l'utilité de ses pièces, puis à le remonter. C'est pourquoi une démarche de type séquentiel peut être tout indiquée dans une telle situation. Par contre, lorsque l'objet est complexe et que les élèves peuvent éprouver de la difficulté à le remonter, la dissection mécanique 
séquentielle n'est pas à privilégier, et cela, puisque les élèves doivent être capables de réassembler l'objet pour en comprendre le fonctionnement.

Lors des entretiens semi-dirigés, quatre élèves (deux équipes) qui ont fait une dissection mécanique de type séquentiel nous ont mentionné qu'ils procèderaient d'une façon différente s'ils étaient de nouveau confrontés à une tâche de dissection mécanique. En fait, ils ont dit avoir entièrement démonté l'objet rapidement sans prendre le temps planifier leur démarche. Voici un exemple de propos tenus :

En premier, on a tout démonté. Après ça, on s'est rendu compte qu'il ne fallait pas tout démonter, on a essayé de remonter. Puis après ça... quand on a eu fini de remonter, bien on a réussi à comprendre le mécanisme en gardant les pièces importantes. (Participant 2)

Lorsque nous avons demandé à cet élève s'il utiliserait la même démarche lors d'une prochaine dissection mécanique, il a mentionné que son coéquipier et lui n'ont pas procédé de façon optimale. Il souligne qu'ils n'auraient pas dû démonter entièrement le diffuseur de fragrance automatique : « on aurait pu juste enlever les parties non importantes » (Participant 2), comme les vis et la coquille extérieure du diffuseur. Ensuite, il met l'accent sur l'importance de la planification pour les prochaines dissections mécaniques : « je commencerais par essayer de trouver un moyen de trouver comment ça marche » (Participant 2).

Par contre, les membres d'une équipe ayant réalisé une dissection mécanique séquentielle ont affirmé vouloir procéder exactement de la même manière la prochaine fois. Ce qu'il faut savoir, c'est que la dissection mécanique d'objet n'était pas nouvelle pour ces deux élèves ; ils ont même démonté un véhicule tout-terrain à la maison.

Lorsque nous avons demandé au participant 12 (qui est membre de cette équipe) s'il avait dû prendre le temps d'analyser le fonctionnement de l'objet lors de la dissection, il a mentionné : «pour quelqu'un qui ne sait pas comment ça fonctionne, certainement, mais comprenant le fait qu'on savait déjà de quelle façon ça marchait... [sous-entendu : ce n'était pas nécessaire] ». En somme, la dissection mécanique proposée dans cette recherche n'était donc pas adéquate pour ces élèves. L'objet étant trop facile à analyser pour eux, ils ont pu employer la dissection mécanique séquentielle sans aucune difficulté.

Bref, deux facteurs ont un impact sur le choix de la démarche à privilégier par les élèves et sa réussite : la complexité de l'objet à démonter et la familiarité des élèves 
avec la dissection mécanique. Autrement dit, un objet trop simple pour les élèves peut les amener à réaliser une dissection mécanique séquentielle sans difficulté, surtout s'ils ont l'habitude de réaliser des dissections mécaniques. Par contre, lorsque la dissection mécanique est un défi (réalisable) pour les élèves, c'est-à-dire d'un niveau de difficulté adéquat pour eux, ce cas de figure n'est pas indiqué. Par conséquent, tout comme Doucet et al. (2007), nous croyons en l'importance pour l'enseignant de sélectionner l'objet à disséquer en fonction de sa complexité et du niveau de ses élèves.

\section{Rôle de la définition de la dissection mécanique dans le choix du cas de figure de la dissection séquentielle}

La définition de la dissection mécanique de Doucet et al. (2007) et celle de Dalrymple (2009) précisent qu'il faut démonter, observer et analyser le fonctionnement de l'objet. Dalrymple (2009) ajoute à ces étapes celle de remonter l'objet. Ces définitions pouvant laisser entendre qu'il s'agit d'un processus linéaire, il nous semble possible qu'elles introduisent un obstacle didactique dans l'apprentissage des élèves, puisque « les obstacles d'origine didactique sont ceux qui sont provoqués par le choix d'enseignement » (Kazadi, 2015, p. 64). Un élève qui lit ou entend cette définition peut l'envisager comme une séquence dans le temps, c'est-à-dire une démarche qui consiste à démonter un objet, puis à l'observer et finalement à l'analyser, ce qui se rapproche de la dissection séquentielle ${ }^{10}$.

Nous avons analysé attentivement les manuels scolaires de science et technologie québécois du secondaire afin de nous pencher sur les manières dont la dissection mécanique est présentée aux élèves, mais en vain. Les manuels québécois abordent surtout des concepts connexes à la dissection mécanique, comme les systèmes de transmission ou de transformation du mouvement, et non la démarche en tant que telle. Par ailleurs, la collection Biosphère en troisième secondaire et Écosphère en quatrième secondaire (publiée par les éditions CEC) offre une définition de l'analyse technologique similaire à celle du PFEQ (MELS, 2007a) : « La démarche technologique d'analyse vise à effectuer l'analyse de la fonction globale d'un objet ainsi que de la fonction

10 Puisqu'il est difficile d'analyser un objet complètement démonté, il est par conséquent nécessaire de remonter l'objet pour mener la tâche à terme. 
de ses sous-systèmes et de ses composantes » (Dubreuil et al., 2008, p. 269). Nous constatons donc que la présentation de la dissection mécanique aux élèves est déléguée à l'enseignante ou l'enseignant, qui se doit donc d'être vigilant lorsqu'il introduit cette démarche afin d'éviter d'introduire cet obstacle didactique.

\section{Recommandations pour l'enseignement de la dissection mécanique}

L'analyse des résultats de cette recherche nous a permis de formuler des recommandations pour l'enseignement de la dissection mécanique au secondaire. Ces recommandations s'adressent autant aux enseignants de science et technologie qu'à tout autre acteur du milieu éducatif qui se sent concerné. La première recommandation est la suivante : dans une optique d'enseignement de méthodes de travail efficaces, 1) nous recommandons aux enseignants et enseignantes en S\&T de prôner la réalisation d'une dissection mécanique en spirale. Nous avons pu constater que les seuls élèves ayant réussi à comprendre le fonctionnement de l'objet sans avoir à le remonter sont ceux qui ont utilisé la dissection mécanique en spirale. De plus, ce cas de figure permet aux élèves d'observer les mouvements réels de l'objet, dans le cas d'un objet comme le diffuseur de fragrance automatique. Par contre, la dissection mécanique en spirale peut ne pas convenir à tous les élèves. Par conséquent, il est important de ne pas rendre l'utilisation de la dissection mécanique en spirale obligatoire. En effet, la dissection mécanique séquentielle peut suffire lorsque les élèves font face à un objet comportant peu de pièces et dont le fonctionnement est facile à comprendre. De plus, 2) nous recommandons d'enseigner la méthode de dissection mécanique par systèmes lorsque l'objectif pédagogique d'une activité, outre la compréhension du fonctionnement de l'objet, consiste à prendre conscience de l'importance d'un système dans un objet; et cela, bien que ce cas de figure exige de remonter l'objet. Par exemple, dans le cas du diffuseur de fragrance automatique, le système de transformation du mouvement est à la base du fonctionnement de l'objet. En effet, il transforme la rotation provenant du moteur en translation bidirectionnelle permettant d'appuyer sur le bouton, éjectant ainsi la fragrance. La troisième recommandation aborde le cas de figure le plus souvent utilisé par les participants et participantes à notre recherche, malgré tout, 3) nous recommandons aux enseignants et enseignantes de ne pas enseigner la dissection mécanique séquentielle. Comme ce type de dissection a une efficacité variable selon la familiarité des élèves 
à réaliser une dissection mécanique et selon la complexité de l'objet pour eux, nous croyons que ce type de dissection mécanique doit être utilisé seulement par les élèves se sentant à l'aise avec cette manière de faire. Il n'est donc pas indiqué de l'enseigner à un groupe complet ou comme la façon de faire à privilégier. Rappelons que deux des trois équipes ayant utilisé ce cas de figure ont mentionné qu'elles n'envisageaient pas de réutiliser cette méthode dans l'avenir. Comme quatrième recommandation, 4) nous suggérons aux enseignants et enseignantes d'adapter le choix de l'objet en fonction du niveau des élèves, tout comme le mentionnent Doucet et al. (2007). Pour un objet plus complexe, l'objet pourrait être disséqué par l'élève avec l'appui de l'enseignant ou d'un pair plus familier avec la dissection mécanique. Ceci rappelle le concept de zone proximale de développement proposé par Vygotski. Il s'agit de la zone cognitive dans laquelle des apprentissages plus complexes pour l'apprenant deviennent possibles avec de l'aide provenant d'un adulte ou d'un pair (Vienneau, 2011). Finalement, rappelons que les diverses définitions de la dissection mécanique peuvent donner une impression de linéarité à cette démarche, alors qu'elle est plutôt itérative. La présentation de la définition peut introduire un obstacle didactique et amener les élèves à utiliser le cas de figure séquentiel. Par conséquent, 5) nous recommandons aux enseignants et enseignantes de formuler avec soin la définition de la dissection mécanique qu'ils fournissent aux élèves.

\section{Conclusion}

En conclusion, cette recherche a permis de décrire de quelles façons des élèves du secondaire en science et technologie (et plus particulièrement dans le profil Applications technologiques et scientifiques) opérationnalisent la dissection mécanique. Trois cas de figure ont émergé, soit la dissection mécanique séquentielle, la dissection mécanique par systèmes et la dissection mécanique en spirale. En raison de la nature exploratoire de cette recherche, nous pouvons affirmer qu'il existe au moins ces trois cas de figure dans la réalisation de la dissection mécanique, mais il importe de garder en tête qu'il en existe possiblement d'autres. Par conséquent, nous ne prétendons pas que les résultats de cette recherche sont exhaustifs. Il serait ainsi pertinent de répéter la recherche détaillée dans cet article avec un plus grand nombre de participants et de participantes, de manière à 
identifier de nouvelles manières d'opérationnaliser la dissection mécanique (s'il y a lieu) et à consigner la répartition de ces participants et participantes entre les différents cas de figure.

Une des limites de notre recherche repose sur le fait que nous ne connaissons pas la façon dont la dissection mécanique a été enseignée aux élèves qui y ont participé. Cela dit, il nous aurait été impossible de retracer le parcours scolaire des élèves alors qu'ils réalisent probablement des démarches d'analyse technologique depuis le début de leur secondaire, et peut-être même depuis le primaire. Nonobstant ces limites, les résultats de cette recherche contribueront à alimenter les réflexions d'enseignants et de formateurs de science et technologie ainsi que des autres acteurs scolaires concernés par l'enseignement de la démarche d'analyse technologique telle qu'elle est décrite par le MELS (2007a). Dans le souci de mieux comprendre la manière de réaliser des dissections mécaniques par des élèves d'une part, et la manière de les enseigner par le personnel scolaire (enseignants et techniciens en travaux pratiques) d'autre part, et dans une optique de clarifier les enjeux didactiques relatifs à l'enseignement et à l'apprentissage des démarches d'analyse technologique, d'autres recherches seraient à envisager. Il pourrait par exemple s'agir de recherches visant à comparer les démarches d'analyse technologique du premier et du second cycle du secondaire ou d'effectuer des études comparatives entre les provinces du Canada, cette recherche se limitant au contexte québécois. Enfin, à la lumière des résultats de cette recherche, nous comprenons mieux les manières dont des élèves de quatrième secondaire procèdent lorsqu'ils font une analyse technologique, une étape préalable à la réparation d'un objet. La suite logique de cette démarche, c'est-à-dire la réparation de l'objet, gagnerait elle aussi à faire l'objet de recherches, d'autant plus qu'elle figure explicitement au programme de formation québécois. 


\section{Références}

Barma, S. et Guilbert, L. (2006). Différentes visions de la culture scientifique et technologique : défis et contraintes pour les enseignants. Dans A. Hasni, Y. Lenoir et J. Lebeaume (dir.), La formation à l'enseignement des sciences et des technologies au secondaire (p. 9-39). Presse de l’Université du Québec.

Chikofsky, E. J. et Cross, J. H. (1990). Reverse engineering and design recovery: A taxonomy. IEEE Software, 7(1), 13-17. https://doi.org/10.1109/52.43044

Doucet, P., Langelier, È. et Samson, G. (2007). Une démarche de conception en sept étapes. 2e partie : la rétro-conception et la dissection mécanique. Spectre, décembre-janvier, 30-33.

Dalrymple, O. O. (2009). The pedagogical value of disassemble/analyze/assemble (DAA) activities: Assessing the potential for motivation and transfer (Publication no UMI 3402322) [Thèse de doctorat, Purdue University]. ProQuest Dissertations and Theses.

Dalrymple, O. O., Sears, D. A. et Evangelou, D. (2011). The motivational and transfer potential of disassemble/analyze/assemble activities. Journal of Engineering Education, 100(4), 741-759. https://doi.org/10.1002/j.2168-9830.2011.tb00034.x

Damphousse, J. (2017). La dissection mécanique réalisée par des élèves du secondaire en sciences et technologie: Démarches employées et sens utilisés [Mémoire de maîtrise, Université du Québec à Trois-Rivières]. Cognitio. http://depot-e.uqtr.ca/ $\underline{\mathrm{id} / \text { eprint } / 8350}$

Dubreuil, M., Leroux, D., Morissette, P., Pagé, M. et Poirier, A. (2008). Écosphère. Éditions CEC.

Elizalde, H., Rivera-Solorio, C. I., Pérez, Y., Morales-Menéndez, R., Orta, P., GuerraZubiaga, D. et Ramírez-Mendoza, R. A. (2008). An educational framework based on collaborative reverse engineering and active learning: A case study. International Journal of Engineering Education, 24(6), 1062-1070.

Fortin, M.-F. et Gagnon, J. (2016). Fondements et étapes du processus de recherche : méthodes quantitatives et qualitatives ( $3 \mathrm{e}$ éd.). Chenelière éducation. 
Gonzalez, D. (2019, 21 mars). Une action collective contre Apple autorisée au Québec. Radio-Canada. https://ici.radio-canada.ca/nouvelle/1159701/iphone-lentobsolescence-programmee-recours-collectif-apple-justice-ios-simon-st-onge

Groleau, A. et Pouliot, C. (2015). Aborder la notion d'obsolescence en classe pour éclairer les aspects économiques et environnementaux de la technologie. Spectre, 44(2), 34-36. https://fr.calameo.com/aestq/read/0051814834ae9c46f8ede

Kazadi, C. (2015). Lexique de didactique des mathématiques. Les Éditions Pythagore.

Latouche, S. (2012). Bon pour la casse : les déraisons de l'obsolescence programmée. Éditions Les Liens qui libèrent.

Ministère de l'Éducation, du Loisir et du Sport [MELS]. (2007a). Programme de formation à l'école québécoise. Enseignement secondaire, Programme d'applications technologiques et scientifiques, 2e cycle. http://www.education. gouv.qc.ca/fileadmin/site_web/documents/education/jeunes/pfeq/PFEQ applications-technologiques-scientifiques.pdf

Ministère de l'Éducation, du Loisir et du Sport [MELS]. (2007b). Programme de formation à l'école québécoise. Enseignement secondaire, Programme de science et environnement, 2 e cycle. http://www.education.gouv.qc.ca/fileadmin/site web/ documents/education/jeunes/pfeq/PFEQ science-environnement.pdf

Ministère de l'Éducation, du Loisir et du Sport [MELS]. (2007c). Programme de formation à l'école québécoise. Enseignement secondaire, Programme de science et technologie de l'environnement, 2e année du 2e cycle du secondaire. http:// www.education.gouv.qc.ca/fileadmin/site web/documents/education/jeunes/pfeq/ PFEQ_science-technologie-environnement.pdf

Ministère de l'Éducation et de l'Enseignement supérieur [MEES]. (2016). Épreuves uniques : enseignement secondaire, 2e cycle [Document d'information]. https:// numerique.banq.qc.ca/patrimoine/details/52327/3298777

Paillé, P. et Mucchielli, A. (2016). L'analyse qualitative en sciences humaines et sociales (4e éd.). Armand Colin.

Paré, I. (2018, 5 novembre). Combattre l'obsolescence programmée, une réparation à la fois. Le Devoir. https://www.ledevoir.com/societe/consommation/540593/ consommation-combattre-1-obsolescence-programmee-une-reparation-a-la-fois 
Sandborn, P., Myers, J., Barron, T. et McCarthy, M. (2009). Using teardown analysis as a vehicle to teach electronic systems manufacturing cost modelling. International Journal of Engineering Education, 25(1), 42-52.

Trudel, P. (2019, 2 avril). L’obsolescence programmée. Le Devoir. https://www.ledevoir. com/opinion/chroniques/551204/1-obsolescence-programmee\#

Vienneau, R. (2011). Apprentissage et enseignement: Théories et pratiques (2e éd.). Gaëtan Morin.

Younis, M. B. et Tutunji, T. (2012). Reverse engineering course at Philadelphia University in Jordan. European Journal of Engineering Education, 37(1), 83-95. https://doi.org/10.1080/03043797.2012.658508 